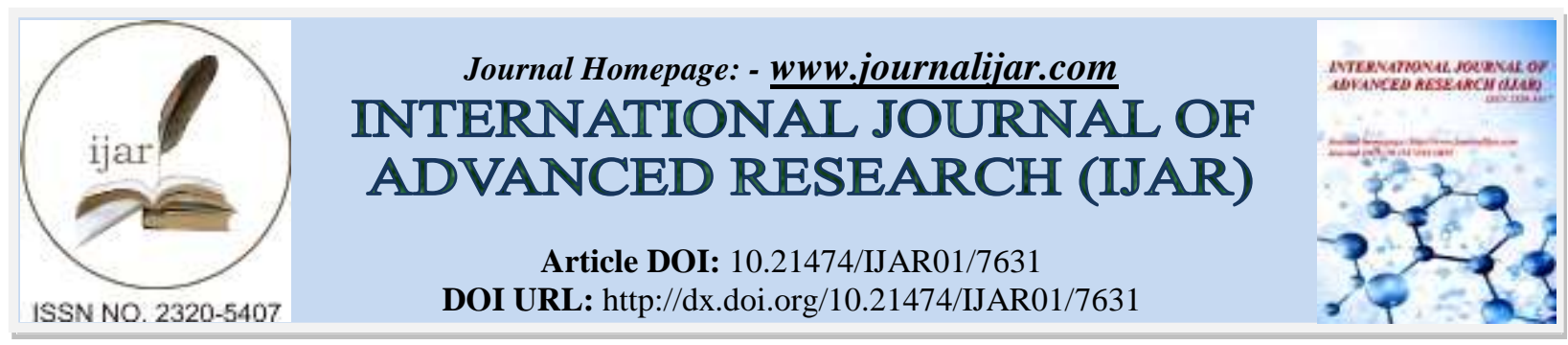

RESEARCH ARTICLE

\title{
DYSLIPIDEMIA AS A CAUSATIVE FACTOR IN HEMORRHAGIC STROKE OF PATIENTS YOUNGER THAN 40 YEARS OF AGE: A PROSPECTIVE STUDY.
}

Vikrant singh Vanita Gupta Jamil-ul-Hussain.

\section{Manuscript Info}

Abstract

Manuscript History

Received: 24 June 2018

Final Accepted: 26 July 2018

Published: August 2018

Copy Right, IJAR, 2018,. All rights reserved.

\section{Introduction:-}

Stroke is broadly defined as abrupt onset of focal or global neurological deficit caused by ischemia or hemorrhage into or around brain, which is irreversible within 24 hours as a result of cerebral blood vessel disease. The resulting neurological syndrome reflects location, size and type of stroke in young is defined as stoke occurring below the age of 40 years [1]. However stroke in young individual poses a major problem as these young men and women are major bread earners of family [2].

The prevalence of stoke is twice that of multiple sclerosis in age group 18 to 40 years. The overall prevalence of cerebro-vascular accidents is 794 per 100,000 populations with male preponderance [3]. Community based surveys from west and Japan indicates average annual incidence of stoke as 111-180 per 100,000 population and 928/100,000 young person [2]. In India Abraham et al [4] from Vellore (South India) reported 25\% cases were less than 40 years of age Bansal et al also reported a high incidence of stroke in young [5].

Many people believe that Asians have life expectancy of less than 60 years and that $50 \%$ of population at any given time is less than 50 years of age, which is responsible for the bias towards the young in the hospital incidence of stroke in young. However it is worth mentioning that life expectancy in some of north east state like Haryana and Punjab is approximately 65 years i.e. almost near western figures, but still the hospital incidence of stroke in young is higher [6].

Frequency of spontaneous intracerebral hemorrhage in young adult varies from $0.7 \%$ to $90 \%$. The etiological spectrum in young adults are wider and include vascular malformation, hypertension and drug use [7, 8]. Various studies (Ueshnia et al 1988, Kangam et al 1980, MRFIT 1989) showed an inverse association between serum cholesterol and cerebral haemorrhage.

\section{Material And Methods:-}

The present study was conducted at Government Medical College Jammu on patients of hemorrhagic stroke Inclusion criteria;

Age 15 to 40 years

Both sexes including pregnant females were included in the study

Diagnosis of stroke was established when Patient had a focal/generalized neurological deficit lasting for 24hours and confirmed by physical finding and computed tomography to be of vascular origin. 
The diagnosis of intracerebral hemorrhage was indicated by following phenomenon

- History of hypertension.

- Rapid evolution of hemiplegia and other phenomenon over minutes or hours

- Onset generally during activity

- Rapid progression to coma

- Symptoms of space occupying lesion e.g. incontinence, headache or vomiting

The controls:

Healthy volunteer < 40years of age of both sexes without any evidence of stroke/ TIA, without any family history of hyperlipidemia, any history of chronic liver disease, chronic renal disease, hypothyroidism were selected to provide control. The subjects were registered after informed consent and eligibility criteria were ascertained. A detailed medical history was obtained from each subject including quantitative information about important risk factors. Routine workup of the subjects was done in hospital wards.

In all patients, the fasting peripheral venous blood sample $(5 \mathrm{ml})$ was taken from each patient using disposable syringe and put in a chemistry tube. After allowing the sample to clot at room temperature for two hours, serum was separated by centrifugation at $1500 \mathrm{xg}$ for 15 minutes at 4 degree Celsius and put into separate vials for storage at 70 degree Celsius (for up to one week)

Commercially available kits from Randox UK were used for estimation of total lipids, cholesterol, LDL Cholesterol and triglycerides.

The data analysis: In addition to descriptive statistics, the $\mathrm{T}$ Test was used to compare between the means of continuous variables.

Observation:-

A total of 37 patients of intracerebral hemorrhage and 50 control subjects were studied. At the end of study data was analysed statistically and observations were as follows

Table no 1:- Showing Sex distribution of patients and controls

\begin{tabular}{|l|l|l|}
\hline & Intracerebral hemorrhage & Control \\
\hline Males & $21(56.75 \%)$ & $35(70 \%)$ \\
\hline Females & $16(43.24 \%)$ & $15(30 \%)$ \\
\hline Total & 37 & 50 \\
\hline
\end{tabular}

Among 37 patients of intracerebral hemorrhage 21 patients i.e. $56.75 \%$ were male patients and sixteen patients i.e. 43.245 were females, where as among the control groups 35 patients $(70 \%)$ were male and fifteen (30\%) were females.

Table no 2:- Showing Age wise representation of patients and controls

\begin{tabular}{|l|l|l|l|l|}
\hline Age group & Intracerebral hemorrhage & Control & Females \\
\hline & Male & Females & Males & 0 \\
\hline $16-20$ yrs & $2(5.4 \%)$ & 0 & $1(2 \%)$ & $1(2 \%)$ \\
\hline $21-25$ yrs & $2(5.4 \%)$ & $1(2.7 \%)$ & $2(4 \%)$ & $6(12 \%)$ \\
\hline $26-30$ yrs & $3(11.11 \%)$ & $6(16.21 \%)$ & $13(26 \%)$ & $1(2 \%)$ \\
\hline $31-35$ yrs & $5(18.51 \%)$ & $5(18.51 \%)$ & $12(24 \%)$ & $7(14 \%)$ \\
\hline $36-40$ years & $9(24.32 \%)$ & $4(14.81 \%)$ & $7(14 \%)$ & $15(30 \%)$ \\
\hline Total & $21(56.75 \%)$ & $16(43.24 \%)$ & $35(70 \%)$ & \\
\hline
\end{tabular}

Our patients as well as controls belonged to the age group of 16 to 40 years. The maximum male patients 9 out of 21 belonged to age group of 36 to 40 years whereas maximum female patients 6 out of 16 belonged to age group of 26 to 30 years. None of these findings was statistically significant. 
Table no 3:- Showing Lipid profile of ICH versus Control

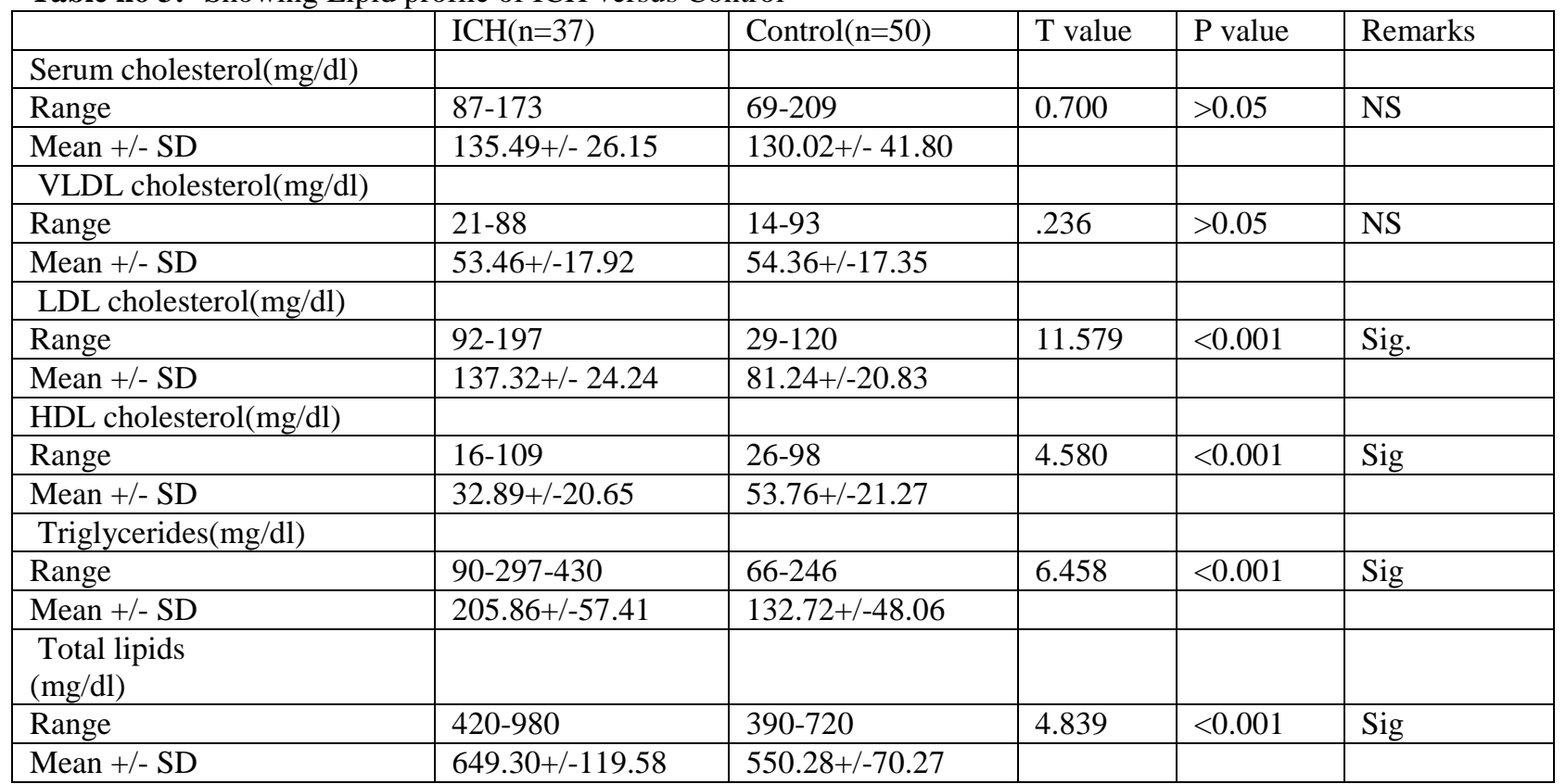

Regarding HDL cholesterol our observation were consistent with observation by Rossner et al, Yatsu and Loch, Venkatraman, Vijayan, Albuchar et al, who observed a significantly lower value of HDL-cholesterol.

The value of triglycerides, LDL-cholesterol and total lipid were statistically significantly higher in patients whereas HDL-cholesterol was statistically significantly lower in patients. The serum cholesterol showed no statistically significant correlation. It was consistent with previously reported by Chopra et al [6] who reported a statistically significant increase in triglycerides and B-lipoproteins. The found a higher values of serum cholesterol which was in contrast to our study.

Our hospital based study could be limited by small number of patients. Larger studies are needed to clarify the status of lipids in various subgroups of patients. We have estimated serum lipids within 48 hours of stroke, although some observers have shown lower value of total cholesterol, LDL-cholesterol and triglyceride and there was no significant effect on HDL- cholesterol and VLDL-cholesterol. But observation of Jacob and Iso [41] who postulated that level of lipid at time of stroke are better representative of lipid levels because poor nutrition or newly developed liver and renal dysfunction after stroke may result in lower lipid levels later on. Also the lipid level measurement at onset of stroke have an advantage of including both fatal and non-fatal stroke. Woo et al [40] reported that acute cerebral hemorrhage has tittle effect on lipid profile and study comprised $70 \%$ patients who were having intracerebral haemorrhage.

Therefore, it seems likely that lipids may be relevant to aetiopathogenesis of cerebrovascular accidents. Large scale studies in human population are needed in defining the exact role of different lipid and lipoprotein in causation and/or progression of cerebrovascular accidents. Rational preventive and/or therapeutic strategies could then be developed as a part of comprehensive primary and secondary prevention of cerebrovascular accidents.

\section{Discussion:-}

Unlike ischemic heart disease, stoke is clinically a more heterogeneous syndrome [12]. Spectrum of etiologies of cerebrovascular accident in young adults is different from older patient and dyslipidemia have been implicated in etiopathogenesis of cerebrovascular accidents. The present study in our institute adds credence to this concept.

In our present study; comparing the lipid profile among $\mathrm{ICH}$ and control group revealed a statistically significant increase in only triglycerides, LDL-cholesterol and total lipids, while cholesterol and VLDL revealed a statistically insignificant difference. In comparison HDL-cholesterol revealed a statistically significant decrease in ICH group $(\mathrm{p}<0.001)$. 
Lipid parameters among intracerebral haemorrhage and ischemic group revealed a statistically significant difference $(<0.001)$ in serum cholesterol being lower in patients of intracerebral hemorrhage, while the difference in other parameter was statistically insignificant $(\mathrm{p}>1.15)$.

Previously reported studies by Joo Louis et al [13] who analyzed risk factors in 200 young stroke patients with intracerebral haemorrhage, found most common risk factors as tobacco use, hypertension, alcohol use and hypocholesterolemia. Hypocholesterolemia with serum cholesterol $<160 \mathrm{mg} / \mathrm{dl}$ was found in about $35 \%$ of patients, multivariate analysis showed hypocholesterolemia more common in patients under age of 20 years (OR-4.87\%.95\% CI, 2.0 to 12.3), there was no significant relationship for triglycerides.

M.Giroud et al (1995) studied risk factors for primary cerebral hemorrhage in 130 cases of intracranial hemorrhage matched with 30 controls residents of Dijon (France). Among quantitative data, the significant factor were early hypertension, high blood glucose, high hematocrit and low cholesterol levels in acute stage of stroke. After multifactorial analysis only two factors were significant; hypertension and low cholesterol levels. This population based study showed that perhaps treatment of hypercholesterolemia may increase risk of intra cerebral hemorrhage.[14]

Philips B. Garelick (1997) stidied about status of lipid as a risk factor for stroke by reviewing previous studies and for cholesterol, they found that studies between 1980 to 1990 generally showed an inverse correlation between cholesterol and cerebral hemorrhage. however one study of MRFIT showed a positive correlation. However three studies in 1990 showed a positive correlation between cholesterol and thromboembolic stroke or cerebral infarction. Also there is an inverse association with HDL, positive association with LDL OR Total Cholesterol and a positive association with triglyceride and apoprotein B [15]

\section{Summary and Conclusions:-}

The present study testifies the important of lipids lipoproteins in cerebrovascular accidents in young adults. It was demonstrated that some abnormalities in lipids and lipoproteins do occur in cerebrovascular accidents in young adults, although exact etiopathogenesis is not known.

Although cause and effect relationship between lipids, lipoproteins and cerebrovascular accident in young adults has not been firmly as yet, the following inference may be safety drawn.

1. In patients of intracerebral haemorrhage, there is an excess of triglyceride, LDL-cholesterol and total lipids while there are low level of HDL-cholesterol in patients. There was no correlation of cholesterol on patients of intracerebral hemorrhage and control, though cholesterol was lower in patient of intracerebral hemorrhage with respect to occlusive stroke.

All said and done the above drawn conclusions are empiric and open to re-examination. In our opinion, there is pressing need to explore vital area of medicine to help in formulation of rational preventive and therapeutic strategies in young stoke patients. As a short term modality we suggest the urgent testing of lipid and lipoprotein in young patients with cerebrovascular accident. 


\section{Bibliography:-}

1. Fischer Marc. Clinical atlas of cerebro-vascular disease- Wolfe revised edition (C) 1994

2. Krutzke J.F. Epidemiology of cerebrovascular disease: Dowel Mc, Capitain LR (eds.) cerebrovascular survey report from national institute of neurologic and communicable disorder and stroke- Bethesda national institute of communicable disease and stroke. 1985

3. Arne Londgaren. Comparison of clinical and neuroradiological finding in first ever stroke - A population based study. Stroke 1994; 25:1371-1377.

4. Abraham J., Rao P.S., Indaraj P.S. An epidemiological study of hemiplegia due to stroke in South India. Stroke 1970(1):977-981.

5. Bansal, B.C., Parkash, C. Cerebrovascular diseases in young individual below the age of 40 years. Neurology India 1973; 21:1-18.

6. Chopra, J.S., Parbhakhar, S. Clinical features and risk factor in stroke in young. Acta Neurology Scan 1979; 60:289-300.

7. Bevan, H., Sharma, K., Bradley, W. Stroke in young adults. Stroke 1990; 21: 382-386.

8. Fuh, J.L., Liu, H.C., Wang, S.J., Lee, L.S. Non traumatic hemorrhagic stroke in young adults in Taiwan. J Stroke cerebrovascular disease 1994; 4:101-105

9. Iso, H., Jacob, Jr. D.R., Wentworth, D., Neaton, J.D., Cohen, J.D. Serum cholesterol and six year mortality from stroke in 350977 men screened for multiple risk factor. NEJM 1989; 20: 904-909.

10. Woo, J., Lam, C.W.K., Kay, R., Wong, H.Y. Acute and longterm changes in serum lipid after stroke. Stroke 1990; 21:1460-1465

11. Parbhakar, S., Chopra, J.S. Stoke in young. Recent concept. Stroke 1999; 45-50.

12. Fuster, V., Kotcke, B.A., Juergan, J.L. Atherosclerosis. Peripheral vascular disease. $5^{\text {th }}$ ed. Philadelphia WB Saunder Co 1980; 219-235.

13. Sandoval, J.M.R., Carlos, C., Fernando, B. Intracerebral hemorrhage in young people. Stroke 1998; 537-541.

14. Girond, M., Creisson, E., Fayelle, H., Andre, N., Becker, F., Martin, D., Dumas, R. Risk factors of primary cerebral hemorrhage -A population based study. A Stroke registry of Dijon. Neuroepidemiology 1995; 14: 2026

15. Philip, B., Gorelick, Michael, S., Lars, F., Berglund, Jerry, G. Status of lipids as a risk factor for stroke. Neuroepidemiology 199; 16: 107-115 